\title{
Diagnostic Dilemma in Hookworm Infection: An Unusual Presentation
}

\author{
Aroop Mohanty*, Priyanka Gupta, Pratima Gupta and Ravi Shankar \\ Department of Microbiology, All India Institute of Medical Sciences, Rishikesh, India \\ *Corresponding author
}

\begin{tabular}{|l|}
\hline Ke y w o r d s \\
Diagnostic \\
Dilemma, \\
Hookworm \\
Infection
\end{tabular}

In this study, a patient who was hospitalized with severe anaemia in the department of Internal Medicine at All India Institute of Medical Sciences, Rishikesh is presented. The patient had fever, swelling of feet since 8 months and cough with expectoration since last 3 months along with significant weight loss three months prior to admission. Severe Iron deficiency anaemia was diagnosed in this patient by laboratory analysis. Patient did not improve despite being transfused with 2 units of packed RBC. Stool examination was performed later as there were no haematological factors associated with severe anaemia. Stool microscopy of the patient revealed numerous ova of hookworm. The general condition improved following anti-parasitic and anti-anaemic treatment. Thus it was concluded that patients diagnosed with iron deficiency anaemia should also be investigated for parasitic diseases which is often neglected.

\section{Introduction}

Hookworm infection is a common public health problem caused by a parasitic nematode that lives in the small intestine of most mammals as its hosts, such as dogs, cats and humans. It is included in the group of Neglected Tropical Diseases (NTD). Approximately one billion people are affected in the underdeveloped countries of the tropics and subtropics. [Hotez et al., 2005] Highest prevalence occurs in sub-Saharan Africa and eastern Asia. Two voracious blood-thirsty nematodespecies namely Ancylostoma duodenale (A.duodenale) and Necator americanus (N.americanus) are responsible for human infection. [Pearson et al., 2010] Worldwide N.americanus is the predominant aetiology of human infection, whereas Ancylostoma duodenale occurs in more scattered focal environments. Infected individuals present with abdominal pain, 
vomiting and iron-deficiency anaemia. Anaemia is the most serious consequence, secondary to loss of iron and protein of the gut. It has been estimated that A.duodenale ingests about $150 \mu \mathrm{L}$ blood per day while N.americanus sucks about $30 \mu \mathrm{L}$. [Midzi et al., 2010] However, this blood loss cannot be visualized by naked eyes. Whereas most of the individuals are asymptomatic, individuals with heavy worm burden, prolonged duration of infection and an inadequate iron intake may manifest with severe iron deficiency anaemia. The diagnosis is based on the identification of characteristic non-bile stained eggs on stool microscopy and eosinophilia on blood examination. [ $\mathrm{Wu}$ et al., 2002] Here we report a case of severe iron deficiency anaemia owing to chronic hookworm (Ancylostoma duodenale) infection.

\section{Case Report}

We hereby report the case of a 62yearoldmale, farmer, resident of Haridwarwas admitted to the Medicine ward with chief complaints of on-off fever since 1 year, swelling of feet since 5 months and cough with expectoration for the last 3 months. He also had difficulty in breathing on walking associated with easy fatigability. On general physical examination, the patient was febrile, tachycardic and tachypenic. Pallor was observedwith bilateral pitting edema and with B/L occasional crepts. Complete blood count revealed haemoglobin $4.5 \mathrm{~g} /$ dland TLC6720/L. Peripheral blood film showed microcytic hypochromic anaemia with no malarial parasite.Liver function tests showed reversal of Albumin/Globulin ratio (2.5/3.6). Blood and urinecultures were sent for and on examination were found to be sterile. Sputum sample was also sent for acid fast bacilli which came negative. Chest X-Ray was done which showed normal findings.. Patient was started on nebulization with bronchodilator and transfused with 2 packs of packed red blood cell. A detailed history was again sought from the patient whereby the patient added passage of blackish stools for the past 8 months. Stool sample was found to be greenish black in colour, solid in consistency and foul smelling without any segments or adult worm. Stool for occult blood was positive. Stool microscopy revealed eggs of hookworm with few pus cells \& RBCs. Stoll's egg counting technique was performed in order to estimate the worm burden. We could find a total of eight hookworm eggs thus resulting in a count of 800 eggs per gram of faeces. As a result of the high load the patient was transfused with 2 units of blood to compensate for severe anaemia. Treatment was initiated with albendazole $400 \mathrm{mg} / 12$ hours for two consecutive days together with oral iron supplements (ferric sulfate) for three months. Two months later all haematological parameters had come back to normal along with a negative examination of faecal parasite.

\section{Results and Discussion}

Hookworm infection is a public health issue in our country. The main risk factors associated with this infection are: living in rural, tropical and subtropical areas, social and economic factors, and poor hygiene, impaired management of biological waste and walking barefoot which enables the penetration of the parasite into the skin. Hookworms live in the small intestine, their eggs are eliminated through human stool and in the appropriate conditions they hatch in the soil to release larvae that mature into ineffective filariform larvae. Infection is acquired by penetration of the $3^{\text {rd }}$ stage larvae (L3) through the skin.L3 migrates through the body into the blood vessels and are carried to the lungs and ultimately to the pharynx, whereby they are swollen and thereby complete their life cycle in the intestine. 
[Hotez, 2008; Roca et al., 2003] Lesions mostly depend on the number of larvae producing the infection, number of migratory larvae and the number of adult parasites developed. Infection is considered severe when there are over 500 parasites. Acute phase is characterized by itching at site of larval penetration, pneumonitis, retrosternal pain, abdominal pain and diarrhoea. On the contrary chronic infection is asymptomatic or manifested by symptoms and signs resulting fromanaemia, which mainly occurs as a result of duodenal ulcers due to infection and the worms feeding on the haemoglobin. This results in iron-deficiency anaemia being one of the most frequently found manifestations of the disease in its chronic phase, just as in our patient. [Roca and Balanzo, 2006] Thus it becomes important to keep hookworm infection as a provisional diagnosis for chronic persistent anaemia not responding to repeated blood transfusions in a rural setting. The diagnosis is established by egg identification in stool examination, so that at least three samples obtained in days are necessary. Since hookworms belong to the order Strongyloidea there may be serological crossed reaction with $S$. stercolaris. Our patient presented negative for $S$. stercoralis.

\section{References}

Hotez PJ, Bethony J, Bottazzi ME, Brooker S, Buss P. Hookworm: 'The great infection of mankind.' PLoS Med 2005; 2:e67.

Hotez PJ. Tropical diseases research: thirty years and counting. PloSNegl Trop Dis.
2008; 2(11):e329.

Martin Sanchez AM, Hernandez Garcia A, Gonzalez Fernanades M, Afonso Rodriguez O, Hernandez Cabrera M, Perez Arellano JL. Parasitosisintestinalesenpoblacioninmig rantesubsaharianaasintomatica. Gran Canaria 2000. Rev ClinEsp 2004; 204(1):14-7.

Midzi N, Mtapuri-Zinyowera S, Mapingure MP, Sangweme D, Chirehwa MT, Brouwer KC, et al., Consequences of polyparasitism on anaemia among primary school children in Zimbabwe. Acta Trop. 2010; 115(1-2):103-111

Pearson MS, Pickering DA, Tribolet L, Cooper L, Mulvenna J, Oliveira LM, et al., Neutralizing antibodies to the hookworm hemoglobinase Na-APR-1: implications for a multivalent vaccine against hookworm infection and schistosomiasis. J Infect Dis. 2010; 201(10):1561-1569.

Roca $\mathrm{C}$, and Balanzo $\mathrm{X}$ Enfermedadesimportadasenimmigrante: mito y realidad. An SistSanit Navar. 2006; 29suppl 1: 139-44.

Roca C, Balanzó X, Sauca G, FernandezRoure JL, Boixeda R, Ballester M. Uncinariasis importada sporinmig rantesafricanos: Estudio de 285 casos. Med Clin. (Barc.). 2003; 121(4): 13941.

Wu KL, Hsu SK, Chiu KW, Chiu YC, Changechien CS. Endoscopic diagnosis of Hooworm Disease of the duodenum: A Case Report J Int Med Taiwan 2002; 13:27-30.

\section{How to cite this article:}

Aroop Mohanty, Priyanka Gupta, Pratima Gupta and Ravi Shankar. 2018. Diagnostic Dilemma in Hookworm Infection: An Unusual Presentation. Int.J.Curr.Microbiol.App.Sci. 7(03): 37693771. doi: https://doi.org/10.20546/ijcmas.2018.703.436 